\title{
PRÁTICAS QUE DIGNIFICAM A PESSOA CUIDADA: PERCEÇÃO DOS ESTUDANTES DE ENFERMAGEM
}

\section{Rosa Cândida Carvalho Pereira de Melo}

Unidade de Investigação em Ciências da Saúde: Enfermagem. Escola Superior de Enfermagem de Coimbra rosamelo@esenfc.pt

\section{Liliana Vanessa Lúcio Henriques}

Unidade de Investigação em Ciências da Saúde: Enfermagem. Escola Superior de Enfermagem de Coimbra lilianahenriques312@gmail.com

\section{Helena Isabel Cardoso da Silva}

Unidade de Investigação em Ciências da Saúde: Enfermagem. Escola Superior de Enfermagem de Coimbra helena-isabel97@hotmail.com

\section{Mónica Andreia Baeta Pereira}

Unidade de Investigação em Ciências da Saúde: Enfermagem. Escola Superior de Enfermagem de Coimbra 4832monicapereira@aefv.edu.pt

Tânia Rodrigues Esteves

Unidade de Investigação em Ciências da Saúde: Enfermagem. Escola Superior de Enfermagem de Coimbra esteves.tania@hotmail.com

Kátia Lilian Sedrez Celich

Universidade Federal da Fronteira Sul - Brasil

elich@uffs.edu.br

\section{RESUMO}

A dignidade humana, que envolve a autonomia e o poder de decisão, é um atributo intrínseco do ser humano que se realiza no encontro com o outro. Esta engloba uma componente relacional que é fundamental para o respeito da pessoa cuidada. Desta forma, importa identificar os significados atribuídos pelos estudantes de enfermagem ao conceito de dignidade e identificar as práticas de cuidados que dignificam a pessoa cuidada. Assim, foi realizado um estudo descritivo transversal, com abordagem qualitativa e quantitativa, realizado a 115 estudantes do $2^{\circ}$ ano do curso de licenciatura de Enfermagem, sendo 93 do sexo feminino (80,8\%), 21 do masculino (18,3\%) e 1 outro (0,9\%), com idades compreendidas entre 18 e 41 anos, com média de 20,61 anos. Os dados foram recolhidos através de um questionário, tendo sido solicitado que respondessem às seguintes ques- 
tões abertas: "Identifique 5 palavras que associa ao conceito de dignidade" e "Quais as práticas que dignificam a pessoa cuidada?". Os dados obtidos foram analisados recorrendo à técnica de análise de conteúdo de Bardin (2016) e IBM SPSS Statistics 20. Os significados que os estudantes mais atribuíram ao conceito de dignidade foram: "Respeito" (18,61\%), "Honra" (4,35\%), "Valores" $(4,17 \%)$, "Integridade" (4,00\%) e "Direito" (3,65\%). No que concerne às práticas que dignificam a pessoa cuidada emergiram 7 categorias: "Respeito" teve maior relevância com 63 indicadores; "Interação" com 43 indicadores; "Autonomia" com 28 indicadores; "Qualidade de vida" com 27 indicadores; "Pilares da Humanitude" com 17 indicadores; "Deontologia" com 15 indicadores; e "Cuidado Centrado na Pessoa" com 12 indicadores. 0 conceito de dignidade apresentou diversas interpretações, dificultando a sua compreensão e consolidação conceptual, existindo a necessidade de promovermos um maior esclarecimento, prevenindo o risco deste conceito essencial, para a práxis do cuidado, vir a ser banalizado.

Palavras-chave: dignidade humana; práticas de cuidados; estudantes de enfermagem

\section{ABSTRACT}

Practices which dignify the person the person: Nursing students' perception. Human dignity, which involves autonomy and decision power, is a human intrinsic attribute which happens in the encounter of each other. This one involves one relational and fundamental respecting the cared person. In this way, it is important to identify the meanings given by the nursing students regarding the dignity concept and identify the care practices which dignify the cared person. In this way, it was created a transversal descriptive study, which qualitative and quantitative approach, done by 115 students from $2^{\circ}$ year of graduation of Nursing, where 93 were females $(80,8 \%)$, 21 were males $(18,3 \%)$ and 1 other gender $(0,9 \%)$, whit ages between 18 and 41 years old, average age is 20,61 years old. The data was collected through a quiz, which has been requested to reply to the following open questions: "Identify 5 words which associate the dignity concept" and "Which practices dignify the cared person?". The data retrieved were analyzed using the analyze technique of the content from Bardin (2016) and IBM SPSS Statistics 20. The meanings identified with more selection from the students were: "Respect" (18,61\%), "Honor" (4,35\%), "Values" (4,17\%), "Integrity" $(4,00 \%)$ and "Right" $(3,65 \%)$. Concerning the answers for the practices which dignify the cared person 7 categories showed up, following "Respect" which had greater relevance with 63 indicators; "Interaction" with 43 indicators; "Autonomy" with 28 indicators; "Life Quality" with 27 indicators; "Human Pillars" with 17 indicators; "Deontology" with 15 indicators; and "Person Centralized Cared" with 12 indicators. The dignity concept presented multiple interpretations, which difficulted the understanding and conceptual consolidation, existing the need to promote a bigger clarification, preventing the risk of this essential concept, for the praxis of care, being banalized.

Keywords: human dignity; care practices; nursing students

\section{INTRODUÇÃo}

A dignidade é um conceito vago, complexo, multidimensional e fulcral para a sociedade civil, denotando-se como central na profissão de Enfermagem. A complexidade contextual reivindicada baseia-se nas inúmeras tentativas de teorização do seu significado, durante a história humana (Parandeh, Khaghanizade, Mohammadi \& Mokhtari-Nouri, 2016). Uma breve análise cronológica do termo permite-nos identificá-lo como proveniente do sânscrito dec, sinónimo de conveniente, adequado, conforme a algo ou alguém. Posteriormente, foi adotada pelas línguas latinas, à qual acrescentaram o sufixo mus, formando o vocábulo decmus, tornando-se em dignus (Vecchi, Garcia \& Sobrinho, 2020), significando algo próximo do valor de honra (Buchanan, 2015). Segundo o mesmo autor, avançando nesta viagem do tempo, a perspetiva dos estoicos, defendia que a dignidade seria 
alcançada se cada indivíduo usasse em pro bono o que separava a humanidade das bestas: a capacidade de raciocinar e viver de forma pensativa e reflexiva (Buchanan, 2015).

Immanuel Kant teve um papel fundamental na definição do conceito de dignidade humana, permanecendo na atualidade como valor moral, com enorme influência a nível ético-jurídico. Este literato subdivide o valor moral em dois tipos: 0 mérito - construído, aprimorado ou diminuído pela pessoa e o inalienável que reitera as perceções e, subsequentes, ações dos outros para com o indivíduo (Buchanan, 2015).

Dignidade foi um dos "valores supremos" (Shultziner, 2003) invocados em documentos base da nossa civilização, como a Carta das Nações Unidas e a Declaração Universal dos Direitos Humanos. Enquanto disciplina, vários especialistas exploraram metaparadigmas de diversos modelos, não concebendo uma teorização única acerca do conceito (Simões \& Sapeta, 2019). Chochinov (2006), referência mundial no estudo da dignidade humana, iniciou a sua investigação sobre a dignidade da pessoa doente em 1995, sugerindo três categorias de questões: (1) illness-related issues, (2) dignity-conserving repertoire e (3) social dignity inventory. Os temas de cada categoria sugeriram 0 Modelo de Dignidade Humana e o movimento dignity in care, que dá orientação terapêutica a profissionais de saúde, ressaltando aspetos físicos, psicológicos, sociais e espirituais/existenciais que podem afetar 0 doente (Buchanan, 2015). Chochinov et al. (2008) apresentaram, subsequentemente, um instrumento que permitiu avaliar o sentimento de dignidade dos pacientes em fim de vida. Posteriormente, a escala foi adaptada para o português, recebendo o nome Escala da Dignidade do Doente, sendo bastante utilizada na prática clínica ao nível dos Cuidados Paliativos.

A dificuldade em compreender 0 conceito de dignidade humana tem constituído um enorme desafio, a profissionais e estudantes de Enfermagem, na argumentação do respeito pela dignidade como central na condução adequada de um cuidado de Enfermagem. Os estudantes de Enfermagem, no decorrer do seu percurso académico, são alertados para a necessidade da compreensão do respeito pela dignidade da pessoa, como elemento central na práxis do cuidado. Todavia, é expectável que os alunos detenham uma débil compreensão conceptual de dignidade. Estas limitações na compreensão e descrição clara do conceito, podem conduzir à sua banalização, quando abordada a temática da ética e deontologia em Enfermagem. Perspetiva-se uma gradual evolução associativa deste termo com o decorrer de ensino clínicos e com compreensão de situações de dano da dignidade da pessoa. Este aspeto central dos cuidados de Enfermagem carece de formação focalizada e dedicação, por parte de estudantes e profissionais (Simões \& Sapeta, 2019). Neste sentido, o ser humano tem 0 direito de receber tratamento, respeito e proteção, independentemente da sua raça, cor, religião ou outra variável socioeconómica. Estes aspetos devem ser considerados transversais aquando do ensino de enfermagem, tornando-se uma necessidade nos processos educativos e formativos da profissão. Assim, há necessidade de conhecer e compreender os referenciais vinculados à dignidade humana, durante a formação técnico-científica e humanista, com vista à promoção de conhecimento que permita aos estudantes identificar os significados do termo e as práticas de cuidados que dignificam a pessoa cuidada (Costa, Medeiros, Martins \& Coutinho, 2018).

\section{OBJETIVOS DA INVESTIGAÇÃO}

Como objetivos desta investigação pretendemos identificar os significados atribuídos pelos estudantes de enfermagem ao conceito de dignidade e, ainda, identificar as práticas de cuidados que dignificam a pessoa cuidada.

\section{PARTICIPANTES}

Neste estudo participaram 115 estudantes do $2^{\circ}$ ano do curso de licenciatura de Enfermagem de uma escola superior de Enfermagem de Portugal, dos quais 93 participantes são do género femi- 
nino (80,8\%), 21 do género masculino (18,3\%) e 1 participante intitulou-se como outro $(0,9 \%)$, com idades compreendidas entre 18 e 41 anos, com média de 20,61 anos.

\section{METODOLOGIA}

Este estudo tem por base uma metodologia descritiva transversal, com abordagem qualitativa e quantitativa. A amostra, constituída por 115 estudantes do $2^{\circ}$ ano do curso de licenciatura de Enfermagem, frequentou o curso complementar de formação "Desafios no cuidar: Como dignificar a pessoa cuidada?", que decorreu entre 25 de janeiro a 5 de fevereiro de 2021 com duração de 20 horas. Os dados foram recolhidos no primeiro dia de formação, para o diagnóstico de necessidades de formação, através de um questionário, no qual os estudantes respondiam às seguintes questões abertas: "Identifique 5 palavras que associa ao conceito de dignidade" e "Quais as práticas que dignificam a pessoa cuidada?". Para a análise dos dados obtidos, recorreu-se à técnica de análise de conteúdo de Bardin (2016), tendo esta sido realizada por três investigadores. Os dados quantitativos foram analisados no software IBM SPSS Statistics 20. Foram excluídas 12 respostas dos estudantes, sobre as práticas que dignificam a pessoa cuidada, por não se adequarem ao objetivo do estudo.

\section{RESULTADOS}

Relativamente à 1 a questão, os estudantes atribuíram 575 significados ao conceito de dignidade. As palavras mais frequentemente mencionadas foram: "Respeito" com um total de 107 respostas $(18,61 \%)$, "Honra" com 25 respostas (4,35\%), "Valores" com 24 respostas $(4,17 \%)$, "Integridade" com 23 respostas $(4,00 \%)$ e "Direito" com 21 respostas (3,65\%). Para facilitar a visualização dos resultados, na Tabela 1, estão apenas os conceitos que foram mencionados cinco ou mais vezes pelos estudantes.

Tabela 1. Palavras associadas pelos estudantes ao conceito de dignidade humana.

\begin{tabular}{|l|l|l|l|l|}
\hline Palavras evocadas & Frequência & Percentagem & $\begin{array}{l}\text { Percentagem } \\
\text { valida }\end{array}$ & $\begin{array}{l}\text { Percentagem } \\
\text { cumulativa }\end{array}$ \\
\hline Respeito & 107 & 18,609 & 18,609 & 89,043 \\
\hline Honra & 25 & 4,348 & 4,348 & 44,696 \\
\hline Valores & 24 & 4,174 & 4,174 & 96,870 \\
\hline Integridade & 23 & 4,000 & 4,000 & 54,609 \\
\hline Direito & 21 & 3,652 & 3,652 & 30,087 \\
\hline Privacidade & 19 & 3,304 & 3,304 & 66,609 \\
\hline Honestidade & 18 & 3,130 & 3,130 & 40,348 \\
\hline Compreensão & 13 & 2,261 & 2,261 & 15,304 \\
\hline Consideração & 13 & 2,261 & 2,261 & 21,217 \\
\hline Decência & 11 & 1,913 & 1,913 & 24,696 \\
\hline Empatia & 11 & 1,913 & 1,913 & 32,696 \\
\hline Reconhecimento & 11 & 1,913 & 1,913 & 70,435 \\
\hline Igualdade & 10 & 1,739 & 1,739 & 48,000 \\
\hline Pessoa & 10 & 1,739 & 1,739 & 61,913 \\
\hline Aceitação & 9 & 1,565 & 1,565 & 1,565 \\
\hline Bem-estar & 9 & 1,565 & 1,565 & 10,087 \\
\hline Atenção & 8 & 1,391 & 1,391 & 5,043 \\
\hline Conforto & 8 & 1,391 & 1,391 & 17,217 \\
\hline
\end{tabular}




\begin{tabular}{|l|l|l|l|l|}
\hline Cuidar & 8 & 1,391 & 1,391 & 22,783 \\
\hline Digno & 7 & 1,217 & 1,217 & 26,435 \\
\hline Moral & 7 & 1,217 & 1,217 & 58,261 \\
\hline Virtude & 7 & 1,217 & 1,217 & 99,304 \\
\hline Amor & 6 & 1,043 & 1,043 & 3,304 \\
\hline Autonomia & 6 & 1,043 & 1,043 & 8,348 \\
\hline Consciência & 6 & 1,043 & 1,043 & 18,609 \\
\hline Justiça & 6 & 1,043 & 1,043 & 55,826 \\
\hline Valorização & 6 & 1,043 & 1,043 & 97,913 \\
\hline Atitude & 5 &, 870 &, 870 & 5,913 \\
\hline Autoestima & 5 &, 870 &, 870 & 7,304 \\
\hline Ética & 5 &, 870 &, 870 & 35,478 \\
\hline Individualidade & 5 &, 870 &, 870 & 50,435 \\
\hline Qualidade de vida & 5 &, 870 &, 870 & 68,522 \\
\hline
\end{tabular}

Fonte: IBM SPSS Statistics 20

No que concerne aos resultados obtidos da análise de conteúdo à questão: "Que práticas dignificam a pessoa cuidada?" emergiram 7 categorias: "Respeito", que teve maior relevância com 63 indicadores; "Interação" com 43 indicadores; "Autonomia" com 28 indicadores; "Qualidade de vida" com 27 indicadores; "Pilares da Humanitude" com 17 indicadores; "Deontologia" com 15 indicadores; e "Cuidado Centrado na Pessoa" com 12 indicadores.

Na categoria do Respeito, emergiram 3 subcategorias: privacidade; pessoa e crenças e valores. Relativamente à privacidade, foi reforçado que 0 cuidador deve promover 0 "respeito pela privacidade da pessoa" (P83). Quanto à pessoa, foi mencionada a importância do "respeito pela pessoa cuidada no seu todo" (P77) e também, no que concerne às crenças e valores, à medida que se vai prestando cuidados devemos ir "respeitando sempre aquilo que são as suas crenças e valores." (P58).

Na categoria da Interação, emergiram 6 subcategorias: comunicação verbal; escuta ativa; presença; comunicação não-verbal; empatia e relação de confiança. Na comunicação verbal, um dos estudantes referiu a importância de "Interagir com a pessoa, questionar sobre os seus sentimentos e seus medos" (P75), tornando o cuidado individualizado e personalizado, demonstrando escuta ativa, "ouvir, perceber as suas necessidades" (P46). Relativamente à subcategoria da presença, os estudantes enfatizaram a importância de "demonstrar que não estamos ali por rotina, mas para a ouvir e ter em conta 0 toque e 0 olhar demonstrando que não temos pressa ou que não somos "agressivos" (P100). Em relação à comunicação não-verbal, os participantes frisaram a relevância de "o carinho e atenção em todos os atos de enfermagem" (P39), sendo necessário "colocar-se no lugar da pessoa cuidada e dando-Ihe atenção" (P10), mantendo empatia com a pessoa cuidada. A relação de confiança é referida pelos estudantes, como "a pesquisa de potenciais fatores de afastamento/proximidade entre o cuidador e a pessoa cuidada de modo que se possa criar uma relação de confiança em que a última se sinta confortável para expor os seus medos e preocupações." (P77).

Relativamente à categoria autonomia, emergiram 2 subcategorias: autodeterminação e independência. Quanto à autodeterminação, esta foi percecionada pelos estudantes como "Toda a prática que atenda a autonomia e tomada de decisão da pessoa nas suas capacidades físicas, psicoemocionais, culturais e espirituais." (P103), e a independência, como "0 importante para que a pessoa cuidada seja dignificada, em qualquer tipo de prática profissional, é sempre a estimulação da sua inde- 


\section{PRÁtICAS QUE DIGNIFICAM A PESSOA CUIDADA: PERCEÇÃO dOS ESTUDANTES DE ENFERMAGEM}

pendência na realização de qualquer tipo de tarefa. Por mínima que seja a independência, 0 utente sentir-se-á de algum modo dignificado, pois consegue atingir/realizar algo por si próprio" (P109).

$\mathrm{Na}$ categoria qualidade de vida, emergiram 3 subcategorias: promoção do bem-estar, práticas que satisfazem as necessidades humanas fundamentais e práticas que promovem 0 autocuidado. Relativamente à promoção do bem-estar, os estudantes assumiram como tal, todas as que têm 0 intuito de "Promover o seu bem-estar físico e psicológico" (P112). Quanto às práticas que satisfazem as necessidades humanas fundamentais, foram referidas pelos estudantes, por exemplo, "As práticas de higiene, alimentação, cuidar da imagem, etc." (P36), e por fim, na subcategoria práticas que promovem 0 autocuidado, os participantes consideraram as práticas que permitissem "...promover 0 autocuidado." (P110).

$\mathrm{Na}$ categoria das práticas relacionadas com os pilares Humanitude, surgiram as seguintes subcategorias: pilares relacionais e verticalidade. No que diz respeito à subcategoria pilares relacionais, foi enfatizada pelos participantes a importância de "estimular o toque terapêutico, manter 0 olhar ao nível dos olhos da pessoa, utilizar corretamente 0 olhar, ter um tom e forma de falar meiga" (P19). Relativamente à verticalidade, foi referida a importância de 0 cuidador "Promover a verticalidade" (P19) da pessoa cuidada.

Quanto à categoria deontologia emergiram 2 subcategorias: sigilo e consentimento informado. Em relação ao sigilo, os participantes enfatizaram a "importância sigilo profissional" (P7) na prática dos cuidados à pessoa e relativamente ao consentimento informado, referiram que era essencial "pedir sempre a autorização à pessoa para a realização de qualquer procedimento" (P11).

Na categoria cuidados centrados na pessoa, emergiram 2 subcategorias: valorização da pessoa e cuidados individualizados. Na valorização da pessoa foi referido pelos estudantes ser essencial "fazer os possíveis para que essa pessoa se sinta como um ser humano e não apenas um objeto de cuidado de alguém." (P49). Em relação aos cuidados individualizados prestados à pessoa cuidada, os participantes referiram como pertinente "Identificar as suas necessidades, respeitar as suas limitações, adequar o cuidado à situação da pessoa” (P21). Na Tabela 2 são apresentadas as categorias e subcategorias das práticas que dignificam a pessoa cuidada, resultante da análise de conteúdo às respostas dos estudantes à questão "Que práticas dignificam a pessoa cuidada?".

Tabela 2. Categorias e Subcategorias das práticas que dignificam a pessoa cuidada

\begin{tabular}{|c|c|c|c|}
\hline Categorias & Subcategorias & $\mathrm{N}^{\circ}$ & adores \\
\hline \multirow{3}{*}{ Respeito } & Privacidade & 33 & \multirow{3}{*}{63} \\
\hline & Pessoa & 23 & \\
\hline & Crenças e Valores & 7 & \\
\hline \multirow{6}{*}{ Interação } & Comunicação Verbal & 11 & \multirow{6}{*}{42} \\
\hline & Escuta Ativa & 8 & \\
\hline & Presença & 7 & \\
\hline & Comunicação não verbal & 6 & \\
\hline & Empatia & 6 & \\
\hline & Relação de Confiança & 4 & \\
\hline \multirow{2}{*}{ Autonomia } & Autodeterminação & 20 & \multirow{2}{*}{28} \\
\hline & Independência & 8 & \\
\hline
\end{tabular}




\begin{tabular}{|c|c|c|c|}
\hline \multirow{3}{*}{ Qualidade de Vida } & Promoção do Bem-Estar & 13 & \multirow{3}{*}{27} \\
\hline & $\begin{array}{l}\text { Práticas que satisfazem as } \\
\text { Necessidades }\end{array}$ & 12 & \\
\hline & $\begin{array}{l}\text { Práticas que promovem o } \\
\text { Autocuidado }\end{array}$ & 2 & \\
\hline \multirow{2}{*}{ Pilares Humanitude } & Pilares Relacionais & 14 & \multirow{2}{*}{17} \\
\hline & Verticalidade & 3 & \\
\hline \multirow{2}{*}{ Deontologia } & Consentimento Informado & 13 & \multirow{2}{*}{15} \\
\hline & Sigilo & 2 & \\
\hline \multirow{2}{*}{$\begin{array}{l}\text { Cuidado centrado na } \\
\text { Pessoa }\end{array}$} & Cuidado Individualizado & 9 & \multirow{2}{*}{12} \\
\hline & Valorização da Pessoa & 3 & \\
\hline
\end{tabular}

\section{DISCUSSÃO}

Os resultados do presente estudo evidenciaram a existência de uma grande heterogeneidade nos significados atribuídos ao conceito Dignidade, no entanto, os termos Respeito, Honra, Valores, Integridade e o Direito foram as expressões que emergiram nas respostas dos estudantes. A multidimensionalidade conceptual encontrada no estudo, vai ao encontro ao que é expectável, atendendo a que o significado do termo se relaciona com conceitos por si só abstratos (respeito, valor, honra, direito e integridade), dificultando a sua operacionalização na práxis (Simões \& Sapeta, 2019). Apesar dos diferentes modelos reconhecerem a centralidade da dignidade para a disciplina de Enfermagem, não há teorização acerca do conceito. Na abordagem humanística, o respeito é considerado central (Simões \& Sapeta, 2019). Também no estudo de Woolhead, Calnan, Dieppe e Tadd (2004) são identificados alguns termos como identidade, autoestima, integridade, confiança, direitos humanos, igualdade, autonomia, independência e controle. Em situações específicas, a dignidade está associada ao respeito, integridade pessoal, empatia e compaixão (Clancy, Simonsen, Lind, Liveng \& Johannessen, 2020), resultados similares foram encontrados nos estudos de Henriques, Melo, Pinto e Silva (2020) e de Silva et al. (2020).

Quanto às práticas que dignificam a pessoa cuidada, o respeito foi a categoria mais evidenciada pelos estudantes, demonstrando congruência com o significado maioritariamente atribuído ao conceito de dignidade. 0 respeito engloba a privacidade, pessoa e, crenças e valores. A preservação da privacidade é uma das práticas que dignificam a pessoa cuidada, tais como o respeito e a decência na forma de cuidar (Matiti \& Trorey, 2008).

Outra prática que dignifica a pessoa cuidada é a interação que se estabelece entre o cuidador e a pessoa cuidada, nomeadamente através da comunicação verbal e não-verbal, escuta ativa, empatia, estar presente e relação de confiança. A comunicação é reconhecida como fundamental para respeito pela dignidade da pessoa cuidada (Walsh \& Kowanko, 2002), demonstrando empatia com a pessoa cuidada (Kagan, 2015), sendo necessária a entrega, a dedicação e 0 empenho para 0 estabelecimento de um laço relacional (Saraiva, 2011). Nesta interação é fundamental estar presente, ou seja, "estar com", o que implica comportamentos como tocar, ser um bom ouvinte e compreender a experiência vivida da pessoa cuidada (Simões, 2020). Um estudo realizado com estudantes de diferentes áreas profissionais relacionadas com a saúde concluiu que, os estudantes de enfermagem são aqueles que apresentam um nível de empatia mais alto, comparativamente com os estudantes do curso de medicina e psicologia, e, consequentemente, apresentam uma sensibilidade emocional maior na relação com o outro, culminando numa relação interpessoal afetiva e positiva com a pessoa cuidada (Matalinares-Calveta, et al., 2019). 


\section{PRÁtICAS QUE DIGNIFICAM A PESSOA CUIDADA: PERCEÇÃO dOS ESTUDANTES DE ENFERMAGEM}

A promoção da autonomia emergiu também como uma prática que dignifica a pessoa cuidada, sendo evidenciada a preservação da autodeterminação e da independência. Segundo Lima (2018), a autonomia está ligada a dois postulados: liberdade e capacidade. A liberdade está diretamente relacionada com o respeito pela tomada de decisão da pessoa, por sua vez, a capacidade relaciona-se com a capacidade da pessoa para tomar decisões perante 0 autocuidado e ser capaz de 0 realizar, situando-se, assim, no campo da independência (Couto, 2012). A preservação da autodeterminação e da independência face às oportunidades no autocuidado consideram-se um ganho em saúde a nível pessoal, contribuindo desta forma para a dignificação da pessoa (Couto, 2012).

A qualidade de vida que emergiu como uma das categorias de práticas que dignificam a pessoa cuidada, inclui a promoção do bem-estar, as práticas que satisfazem as necessidades humanas fundamentais e as práticas que promovem o autocuidado. Segundo Queirós (2012), a qualidade de vida pode ser obtida através de aspetos mais subjetivos do bem-estar. Esta subjetividade é garantida pelo desenvolvimento da prática de enfermagem, na construção de uma relação de confiança, permitindo ao cuidador providenciar à pessoa um estado de conforto, que promova a sua dignidade (Bacalhau, 2014).

Os pilares Humanitude foram identificados pelos estudantes como uma outra categoria importante para a dignificação da pessoa cuidada. 0 olhar, a palavra e o toque, constituem os pilares relacionais da Metodologia de Cuidado Humanitude, essenciais para estabelecer uma relação positiva com 0 outro (Henriques, 2017). Desta forma, através da utilização dos pilares desta Metodologia, é alcançável a promoção da dignidade humana e a preservação do valor humano (Salgueiro, 2014).

Os estudantes identificaram a preservação do sigilo e do consentimento informado como práticas que dignificam a pessoa cuidada, tendo sido aglomeradas na categoria deontologia. No estudo de Matiti e Trorey (2008), a confidencialidade constitui uma das práticas que contribuem para a preservação da dignidade. Além disso, a prática do consentimento informado é algo que o enfermeiro deve promover à pessoa, estando vinculado no código deontológico do enfermeiro, uma vez que este deve exercer a sua profissão com respeito pela vida humana e a dignidade da pessoa cuidada (Assembleia da República, 2015).

Por último, relativamente aos cuidados centrados na pessoa que inclui a valorização da mesma e cuidados individualizados, Chochinov et al. (2002), reforça que, as estratégias para a valorização da pessoa, apesar das suas dependências e fragilidades, são essenciais para a manutenção da dignidade. Desta forma, é possível providenciar um cuidado personalizado à pessoa cuidada, sendo este primordial para a sua realização pessoal, dignificando-a (Cruz, 2014).

\section{CONCLUSÃO}

Os estudantes que participaram no estudo atribuíram um vasto conjunto de significados ao conceito de dignidade, evidenciando a dispersão e dificuldade na definição do conceito de dignidade humana, demonstrando que os estudantes de enfermagem não apresentam um conhecimento consolidado acerca desta temática. Todavia, alguns termos foram evocados com mais frequência como respeito, honra, valores, integridade e direito. As práticas identificadas pelos estudantes como dignificantes da pessoa cuidada, assentam no respeito, na interação, na autonomia, na qualidade de vida, nos pilares da Humanitude, na deontologia e no cuidado centrado na pessoa.

0 facto de durante o percurso académico não existir formação especificamente direcionada para as práticas que dignificam a pessoa cuidada, pode ajudar a explicar a dificuldade na reflexão sobre a forma como podem garantir o respeito pela dignidade das pessoas que cuidam.

Este estudo possibilitou uma reflexão sobre a importância da inclusão de programas formativos ao longo do percurso académico dos estudantes, centrados no respeito pela dignidade da pessoa 
cuidada, com 0 intuito de alcançar a máxima qualidade dos cuidados prestados e a excelência dos profissionais de enfermagem.

\section{REFERÊNCIAS BIBLIOGRÁFICAS}

Bacalhau, L. (2014). Cuidar centrado na dignidade (Dissertação de Mestrado). Universidade Católica Portuguesa, Instituto de Ciências da Saúde, Portugal. Recuperado de https://repositorio.ucp.pt/bitstream/10400.14/16385/1/Mestrado\%20-\%20CUIDAR\%20CENTRAD0\%20NA\%20DIGNIDADE\%20final.pdf

Bardin, L. (2016). Análise de conteúdo. Lisboa, Portugal

Buchanan, A. (2015). Respect for dignity and forensic psychiatry. International Journal of Law and Psychiatry, 41, 12-17. doi: 10.1016/j.ijlp.2015.03.002

Chochinov, H.M. (2006). Dying, dignity, and new horizons in palliative end-of-life care. CA: A Cancer Journal for Clinicians, 56(2), 84-103. doi: 10.3322/canjclin.56.2.84

Chochinov, H. M., Hassard, T., McClement, S., Hack, T., Kristjanson, L. J., Harlos, M., Sinclair, S. \& Murray, A. (2008). The patient dignity inventory: A novel way of measuring dignity-related distress in palliative care. Journal of Pain and Symptom Management, 36(6), 559-571. doi: 10.1016/j.jpainsymman.2007.12.018

Clancy, A., Simonsen, N., Lind, J., Liveng, A., \& Johannessen, A. (2020). The meaning of dignity for older adults: A meta-synthesis. Nursing Ethics, XX(X), 1-17. doi:10.1177/0969733020928134

Costa, R., Medeiros, S., Martins, J. \& Coutinho, V. (2018). A simulação no ensino de enfermagem: Reflexões e justificativas a luz da bioética e dos direitos humanos. Acta Bioethica, 24(1), 31-38. doi: 10.4067/S1726-569X2018000100031

Couto, G. (2012). Autonomia/Independência no autocuidado sensibilidade aos cuidados de enfermagem de reabilitação (Dissertação de Mestrado). Escola Superior de Enfermagem do Porto, Portugal. Recuperado de https://core.ac.uk/download/pdf/62698318.pdf

Cruz, S. (2014). A dignidade em lares de idosos (Dissertação de Mestrado). Instituto Superior de Serviço Social do Porto, Portugal. Recuperado de https://comum.rcaap.pt/bitstream/ 10400.26/6461/1/S\%c3\%b3nia\%20Alexandra\%20Barros\%20Cruz.pdf

Henriques, L. (2017). Implementação da metodologia de cuidado humanitude numa unidade de cuidados continuados: Contributo para os profissionais e pessoas cuidadas (Dissertação de Mestrado). Universidade de Coimbra, Faculdade de Medicina da Universidade de Coimbra, Portugal. Recuperado de https://estudogeral.sib.uc.pt/bitstream/10316/81425/ 1/LilianaVanessaLucioHenriques.pdf

Henriques, L., Melo, R., Pinto, I. \& Silva, H. (2020). Práticas de cuidados que dignificam a pessoa idosa. In livro de atas do Ageing Congresso 2020, 0 Envelhecimento como um todo, realizado no dia 22 de setembro de 2020.

Kagan, S. (2015). Dignity, evidence, and empathy. Geriatric Nursing 36(5), 394-396. doi: 10.1016/j.gerinurse.2015.08.005

Lei nำ 156/2015 de 16 de setembro. Diário da República nº 181, 1a Série. Assembleia da República. Lisboa, Portugal.

Lima, A. (2018). Dignidade da pessoa humana e autonomia da vontade: Um estudo interdisciplinar sobre os limites éticos e jurídicos nos casos de eutanásia (Dissertação de Mestrado). Universidade Católica do Rio Grande do Sul, Escola de Direito da Pontifícia, Brasil. Recuperado de http://tede2.pucrs.br/tede2/bitstream/tede/8116/2/Andrei_Ferreira_de_Arau\%cc\%81jo_ Lima_DIS.pdf 


\section{PRÁTICAS QUE DIGNIFICAM A PESSOA CUIDADA: PERCEÇÃO DOS ESTUDANTES DE ENFERMAGEM}

Matalinares-Calvet, M. L., Díaz-Acosta, A. G., Rivas-Díaz, L. H., Arenas-Iparraguirre, C. A., BacaRomero, D., Raymundo-Villalva, 0. \& Rodas-Vera, N. (2018). Dysfunctional parenting styles, empathy and socio-demographic variables in nursing, human medicine, and psychology students. Acta Colombiana de Psicología, 22(2), 112-124. doi: 10.14718/acp.2019.22.2.6

Matiti, M. R., \& Trorey, G. M. (2008). Patients' expectations of the maintenance of their dignity. Journal of Clinical Nursing, 17(20), 2709-2717. doi:10.1111/j.1365-2702.2008.02365.x

Parandeh A., Khaghanizade M., Mohammadi E. \& Mokhtari-Nouri J. (2016). Nurses' human dignity in education and practice: An integrated literature review. Iranian Journal of Nursing and Midwifery Research, 21(1), 1-8. doi: 10.4103/1735-9066.174750

Queirós, P. (2012). 0 bem-estar na perspetiva de enfermagem. Série Monográfica Educação e Investigação em Saúde - Enfermagem: de Nightingale aos dias de hoje 100 anos, 89-118. Recuperado de https://www.researchgate.net/publication/268220826_0_BEM-ESTAR_ NA_PERSPETIVA_DE_ENFERMAGEM

Salgueiro, N. (2014). Humanitude - um imperativo do nosso tempo. Coimbra: IGM Portugal Humanitude Ltd. Recuperado de https://issuu.com/joaoaraujo35/docs/humanitude-issuu

Saraiva, D. (2011). 0 olhar dos e pelos cuidadores: Os impactos de cuidar e a importância do apoio ao cuidador (Tese de Mestrado). Universidade de Coimbra, Faculdade de Psicologia e Ciências da Educação e Faculdade de Economia, Portugal. Recuperado de https://estudogeral.sib.uc.pt/bitstream/10316/17858/1/TESE_MISIE_SET2011.pdf

Shultziner, D. (2003). Human dignity - Functions and meanings. Global Jurist, 3(3), 1-21. doi: $10.2202 / 1535-167 X .1110$

Silva, H., Mendes, A., Ferreira, J., Pinto, I., Lagadoiro, A., Martins, A., Henriques, L. \& Melo, R. (2020). Respect for the dignity of the cared person: Meaning assigned by nursing students. In livro de atas do 24th international Nursing Research Conference, Nursing, and training of professionals, realizado entre os dias 9 e 13 de novembro de 2020.

Simões, A. (2020). Estar presente em tempos de pandemia. Reflexão crítica do conceito de presença em enfermagem. International Journal of Developmental and Educational Psychology, 2: 23-32

Simões, A. \& Sapeta, P. (2019). Conceito de dignidade na enfermagem: Análise teórica da ética do cuidado. Revista Bioética, 27(2), 1-9. doi:10.1590/1983-80422019272306

Vecchi, I. D., Garcia, M. L. \& Sobrinho, L. L. P. (2020). 0 Princípio da dignidade humana e suas projeções no âmbito laboral: Possibilidades e limites. Seqüência, 85, 249-286. doi: 10.5007/21777055.2020v41n85p249

Walsh, K., Kowanko, I. (2002). Nurses and patients perceptions of dignity. Journal of Advanced Nursing 8, 143-151. doi: 10.1046/j.1440-172x.2002.00355.x

Woolhead, G., Calnan, M., Dieppe, P., \& Tadd, W. (2004). Dignity in older age: what do older people in the United Kingdom think? Age and Ageing, 33(2), 165-170. doi:10.1093/ageing/afh045 